\title{
Sulfurized Cyclopentadienyl Nanocomposites for Shuttle-Free Room-Temperature Sodium-Sulfur Batteries
}

Carina Yi Jing Lim, ${ }^{a, b \dagger}$ Alex Yong Sheng Eng, ${ }^{a \dagger}$ Albertus D. Handoko, ${ }^{a}$ Raymond Horia, ${ }^{a, b}$ Zhi Wei Seh ${ }^{a^{*}}$

${ }^{a}$ Institute of Materials Research and Engineering, Agency for Science, Technology and Research (A*STAR), 2 Fusionopolis Way, Innovis, Singapore 138634, Singapore

${ }^{b}$ School of Materials Science and Engineering, Nanyang Technological University, 50 Nanyang Avenue, Singapore 639798, Singapore

${ }^{\dagger}$ These authors contributed equally to this work

* Email:sehzw@imre.a-star.edu.sg

\section{Supporting Information}

This file includes seven sections.

\section{Experimental}

\subsection{Materials}

Sodium cubes $(99.9 \%)$, elemental sulfur ( $\geq 99.5 \%)$, ferrocene (bis( $\eta 5$-cyclopentadienyl)iron), sodium chloride, pyrite-type iron disulfide, cyclopentadienyllithium, polyacrylic acid (average Mw: 450,000), carbon black (Super P), sodium perchlorate ( $\geq 98.0 \%$ ), tin (II) chloride, lithium hexafluorophosphate, lithium bis(trifluoromethanesulfonyl)imide and anhydrous solvents: fluoroethylene carbonate, ethylene carbonate, diethyl carbonate, dimethyl carbonate, 1,3- 
dioxolane and 1,2-dimethoxyethane were obtained from Sigma Aldrich and used as received. All electrolyte chemicals and cyclopentadienyllithium were stored and used within an argon-filled glovebox atmosphere $\left(\mathrm{H}_{2} \mathrm{O}<0.1 \mathrm{ppm}, \mathrm{O}_{2}<1 \mathrm{ppm}\right)$.

\subsection{Synthesis of amorphous sulfurized cyclopentadienyl composites}

Sulfurized cyclopentadienyl composites (SCC) were synthesized by two different methods, namely a mixed-phase method and a co-melting method ${ }^{1}$.

a)

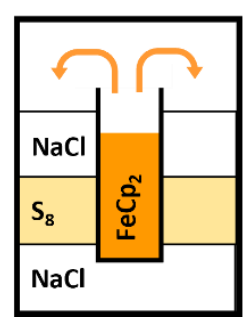

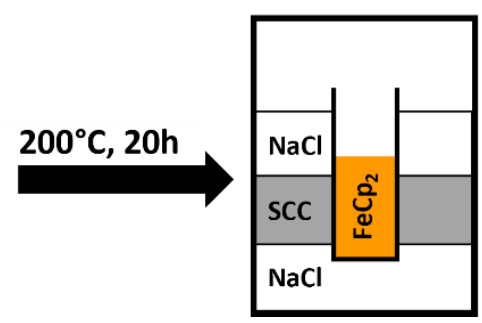

b)

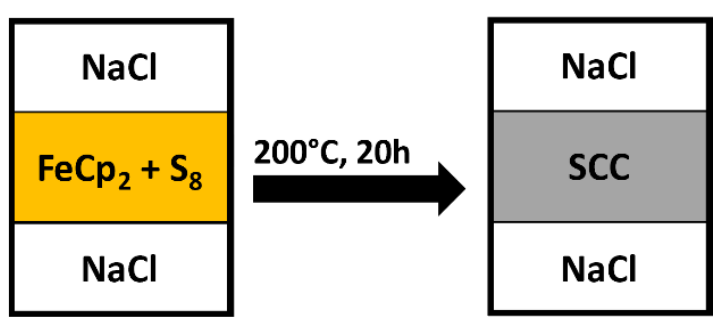

Figure S1. Schematic representation of synthesis set-up via a) mixed-phase method and b) comelting method.

For the mixed phase synthesis (Figure S1a), the sulfur and ferrocene precursors were initially physically separated. Ferrocene (330 mg and $659 \mathrm{mg}$ respectively, for Cp:S ratios of 1:3 and 2:3) was contained in a $4 \mathrm{~mL}$ glass vial and was placed in a Teflon-lined stainless-steel autoclave. Sodium chloride (5 g total) and elemental sulfur $(341 \mathrm{mg})$ was layered around the glass vial and the autoclaves were sealed in an Argon-filled glovebox. Unlike the co-melting method whereby varying the $\mathrm{Cp}: \mathrm{S}$ ratio significantly altered the composition of the products (Table $\mathrm{S} 1$ ), the products obtained by this method is unaffected by the precursor ratios. Based on thermodynamics, the Clausius-Clapeyron equation connotes that the saturation vapor pressure of a material is primarily dependent on the material itself and its temperature. ${ }^{2}$ As such, at a fixed reaction temperature, varying the amount of $\mathrm{FeCp}_{2}$ used does not significantly affect the composite, as the saturated vapor pressure of gaseous $\mathrm{FeCp}_{2}$ remains largely unaffected. This was verified experimentally, where composites synthesized with different $\mathrm{Cp}: \mathrm{S}$ ratios, with the same mass of sulfur and temperature, had negligible differences in their compositions (Table S1). The purpose of the powdered sodium chloride is to serve as a fine template to prevent aggregation of the SCC 
composite, without which the molten-state SCC composite would form a hard rock-like solid on cooling to room temperature, and a particulate composite cannot be obtained.

For the co-melting synthesis (Figure $\mathrm{S} 1 \mathrm{~b})$, bulk elemental sulfur $(\mathrm{S})$ and ferrocene $(\mathrm{FeCp} 2)$ solid were initially mixed by physical grinding with a pestle and mortar in Cp:S mole ratios of 1:3 and $2: 3$ to yield a finely dispersed yellow-orange powdered mixture. In the first heating step, $4 \mathrm{~g}$ of the sulfur-ferrocene mixture was transferred into stainless steel autoclaves and sealed inside an Argonfilled glovebox. The powdered precursor mixtures were sandwiched in between two compacted layers of finely divided sodium chloride salt.

For both methods, each autoclave was heated to $200{ }^{\circ} \mathrm{C}$ from room temperature and held for 20 hours before being allowed to cool naturally. The carbonized solids were ground, washed repeatedly with deionized water and ethanol with centrifugation to remove unreacted precursors and the sodium chloride template, and dried overnight at $70{ }^{\circ} \mathrm{C}$. For the MFSCC, any potential lithium polysulfide side products are hydrolyzed upon contact with water and is removed during the washing step, leaving a composite of predominantly carbon and sulfur. Subsequently in the second step, the black carbonized powders were transferred to alumina boats and placed in a tube furnace (Argon-flow rate of $200 \mathrm{sccm}$, heating rate of $10{ }^{\circ} \mathrm{C} \mathrm{min}{ }^{-1}$ ) maintained at $250{ }^{\circ} \mathrm{C}$ for 3 hours for removal of any excess unreacted sulfur and/or ferrocene. The final product was allowed to cool and subsequently collected as a black powder.

For the metal-free composites (MFSCC), cyclopentadienyllithium was used in place of ferrocene using the same procedure at cyclopentadiene:sulfur $(\mathrm{Cp}: \mathrm{S})$ molar ratios of 1:3 and 1:6. 


\subsection{Materials characterization}

Fourier transform infrared (FTIR) spectrums of the samples were obtained on a Perkin Elmer Spectrum 2000 instrument in transmittance mode. The X-ray diffraction were measured using Bruker D8 Discover goniometer equipped with a capillary focused $\mathrm{I} \mu \mathrm{S} 2.0 \mathrm{Cu} \mathrm{K} \alpha \mathrm{X}$-ray source $(50 \mathrm{kV}, 1000 \mu \mathrm{A})$ and $1-\mathrm{mm}$ pinhole collimator. Vantec 500 with Xe microgap positioned at 420 $\mathrm{mm}$ radius was used as a $2 \mathrm{D}$ area detector and sample height ( $\mathrm{z}$ ) is calibrated using a precision micro-positioner and a laser focusing system. The resulting 2D diffractograms were processed, background corrected and translated to 1D curve using DIFFRAC.EVA software. Thermo Scientific Flash 2000 analyzer was used for elemental CHNS analysis, where samples were prepared in tin-foil capsules. Before each elemental CHNS measurement, sulphanilamide (Elemental Microanalysis, UK) was used as an analytical standard for instrument calibration. Scanning electron microscopy (SEM) images were acquired on a JEOL 7600F field emission scanning electron microscope (JEOL, Japan), with energy dispersive X-ray (EDX) spectroscopy (Oxford Technologies, UK). Raman spectroscopy (WITEC CRM200 Raman System) was conducted with a $532 \mathrm{~nm}$ laser. Given the air- and moisture-sensitive nature of the electrode components, the components for post-mortem analysis were prepared in an argon-filled glovebox and sealed in an air-tight container prior to transport to the loading chamber. EDX elemental mapping and quantification were performed at an accelerating voltage of $15 \mathrm{kV}$. X-ray photoelectron spectroscopy (XPS) was performed on a Thermo-Scientific Theta Probe spectrometer fitted with an $\mathrm{Al} \mathrm{K} \alpha \mathrm{X}$-ray source and all spectra were calibrated to the graphitic carbon peak. Post-mortem ex situ XPS was performed on cycled cathodes using a custom-built airtight holder for direct transfer to the analysis chamber. Cells were cycled galvanostatically at $0.2 \mathrm{C}$ followed by constant voltage application at the stated potential, held until the current decayed to $10 \%$ of the initial current. Time-of-flight secondary ion mass spectrometry (TOF-SIMS) measurements utilized a Bismuth primary ion bean at $30 \mathrm{keV}$, over a 100 x $100 \mu \mathrm{m}$ area, using a TOF.SIMS 5 instrument from IONTOF, Germany. 


\subsection{Electrical and electrochemical characterization}

The cathode slurry, comprising of $70 \mathrm{wt} . \%$ active material, $20 \mathrm{wt} . \%$ carbon black (Super P) and $10 \mathrm{wt} . \%$ polyacrylic acid binder with $\mathrm{N}$-methyl-2-pyrrolidone as the medium, was cast on carboncoated aluminum foil via doctor blade casting and dried at $70{ }^{\circ} \mathrm{C}$. The prepared cathode sheets were then cut into discs of $11.28 \mathrm{~mm}$ diameter, with the areal mass loadings of the composites at approximately $1.3-1.9 \mathrm{mg} \mathrm{cm}^{-2}$ (sulfur loading of $0.5-0.8 \mathrm{mg} \mathrm{cm}^{-2}$ ). All electrochemical tests were conducted using 2032-type coin cells assembled in an argon-filled glovebox $\left(\mathrm{H}_{2} \mathrm{O}<0.1 \mathrm{ppm}, \mathrm{O}_{2}\right.$ $<1 \mathrm{ppm})$. The sodium anode was prepared by freshly cutting off the oxide layer from sodium blocks (99.9\%), rolling the metal into sheets and subsequently cutting it into circular discs (12.7 $\mathrm{mm}$ diameter). For the sodium cells, a glass fiber membrane $(16.2 \mathrm{~mm})$ was used as the separator with $80 \mu \mathrm{L}$ of $1 \mathrm{M}$ sodium perchlorate $\left(\mathrm{NaClO}_{4}\right)$ electrolyte in a 1:1 volume mixture of ethylene carbonate (EC) and dimethyl carbonate (DMC) containing $40 \mathrm{mM}$ tin chloride $\left(\mathrm{SnCl}_{2}\right)$ and 8 vol.\% fluoroethylene carbonate (FEC) additives. For lithium cells, lithium metal discs served as the anode $(16.2 \mathrm{~mm}$ diameter) with a Celgard membrane $(16.2 \mathrm{~mm})$ as the separator. The carbonatebased electrolyte for the lithium cells was $1 \mathrm{M}$ lithium hexafluorophosphate $\left(\mathrm{LiPF}_{6}\right)$ electrolyte in a 1:1 volume mixture of ethylene carbonate (EC) and diethyl carbonate (DEC), while the etherbased electrolyte used was $1 \mathrm{M}$ lithium bis(trifluoromethanesulfonyl)imide (LiTFSI) electrolyte in a 1:1 volume mixture of 1,3-dioxolane (DOL) and 1,2-dimethoxyethane (DME). $25 \mu \mathrm{L}$ of either electrolyte was used for each Li cell.

A battery tester (Neware CT-3008) was used for galvanostatic charge/discharge cycling at varying C-rates $\left(1 \mathrm{C}=1673 \mathrm{~mA} \mathrm{~g}^{-1}\right)$ between $0.6 \mathrm{~V}$ and $2.6 \mathrm{~V} v s . \mathrm{Na} / \mathrm{Na}^{+}$for $\mathrm{NaSB}$, and between $1.0 \mathrm{~V}$ and $3.0 \mathrm{~V} v$ s. $\mathrm{Li} / \mathrm{Li}^{+}$for $\mathrm{LiSB}$. The overpotential between galvanostatic charge/discharge was determined at half of the $2^{\text {nd }}$ cycle discharge capacity. Voltammetric tests were conducted on a Gamry Interface 1010E potentiostat and Tafel analysis was performed on cyclic voltammograms at a scan rate of $0.05 \mathrm{mV} \mathrm{s}^{-1}$. 


\section{Chemical compositions of SCC and MFSCC}

Table S1. Elemental compositions of sulfurized cyclopentadienyl composites synthesised with different conditions acquired from CHNS combustion analysis.

\begin{tabular}{lllllll}
\hline \multirow{2}{*}{ Sample } & \multirow{2}{*}{$\begin{array}{l}\text { Synthesis } \\
\text { Method }\end{array}$} & \multirow{2}{*}{$\begin{array}{l}\text { Cp:S } \\
\text { Ratio }\end{array}$} & & \multicolumn{4}{c}{ Elemental Composition (wt. \%) } \\
\cline { 5 - 7 } & & & $\mathbf{C}$ & $\mathbf{N}$ & $\mathbf{H}$ & $\mathbf{S}$ \\
\hline SCC & Mixed-phase & $2: 3$ & 27.3 & 0.0 & 0.6 & $\mathbf{5 7 . 0}$ \\
SCC & Mixed-phase & $1: 3$ & 27.3 & 0.0 & 0.9 & $\mathbf{5 7 . 2}$ \\
MFSCC & Mixed-phase & $1: 3$ & 25.0 & 0.0 & 1.0 & $\mathbf{6 1 . 9}$ \\
SCC & Co-melt & $2: 3$ & 41.2 & 0.0 & 2.4 & $\mathbf{2 9 . 8}$ \\
SCC & Co-melt & $1: 3$ & 36.5 & 0.0 & 1.7 & $\mathbf{4 0 . 5}$ \\
\hline
\end{tabular}

a)
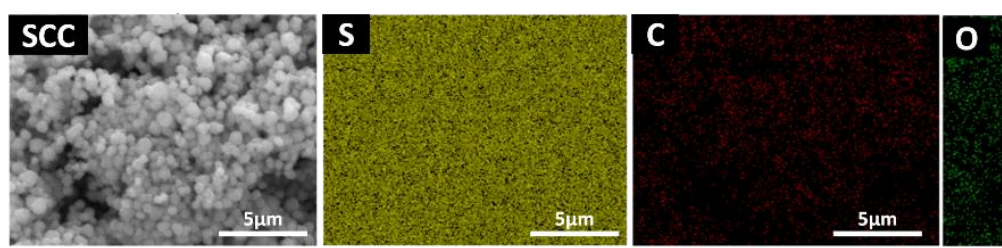

b)
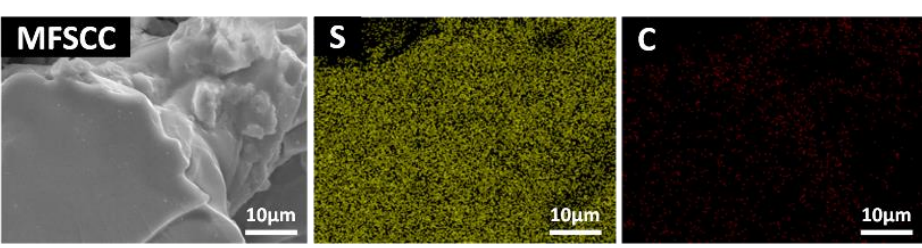
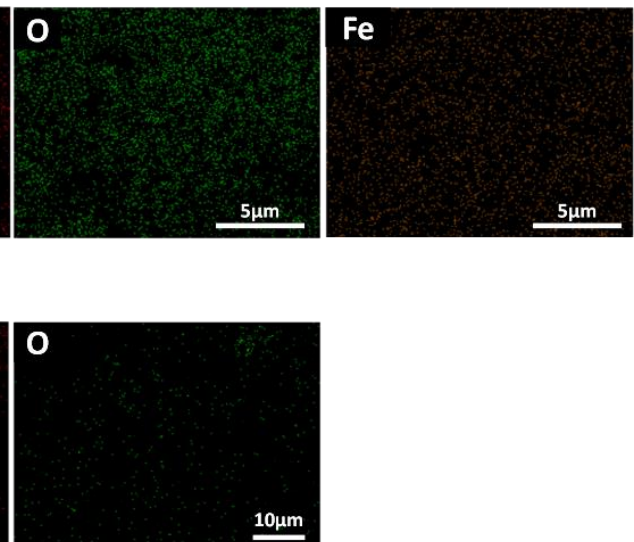

Figure S2. SEM images and corresponding EDX maps of a) SCC and b) MFSCC particulates.

Table S2. Elemental compositions of SCC and MFSCC from EDX spectroscopy.

\begin{tabular}{llllll}
\hline \multirow{2}{*}{ Sample } & \multicolumn{5}{c}{ Elemental Composition (at. \%) } \\
\cline { 2 - 6 } & $\mathbf{C}$ & $\mathbf{N}$ & $\mathbf{O}$ & $\mathbf{S}$ & $\mathbf{F e}$ \\
\hline SCC & 58.9 & 0.0 & 13.0 & 25.4 & 2.7 \\
MFSCC & 72.0 & 0.0 & 1.6 & 26.4 & 0.0 \\
\hline
\end{tabular}



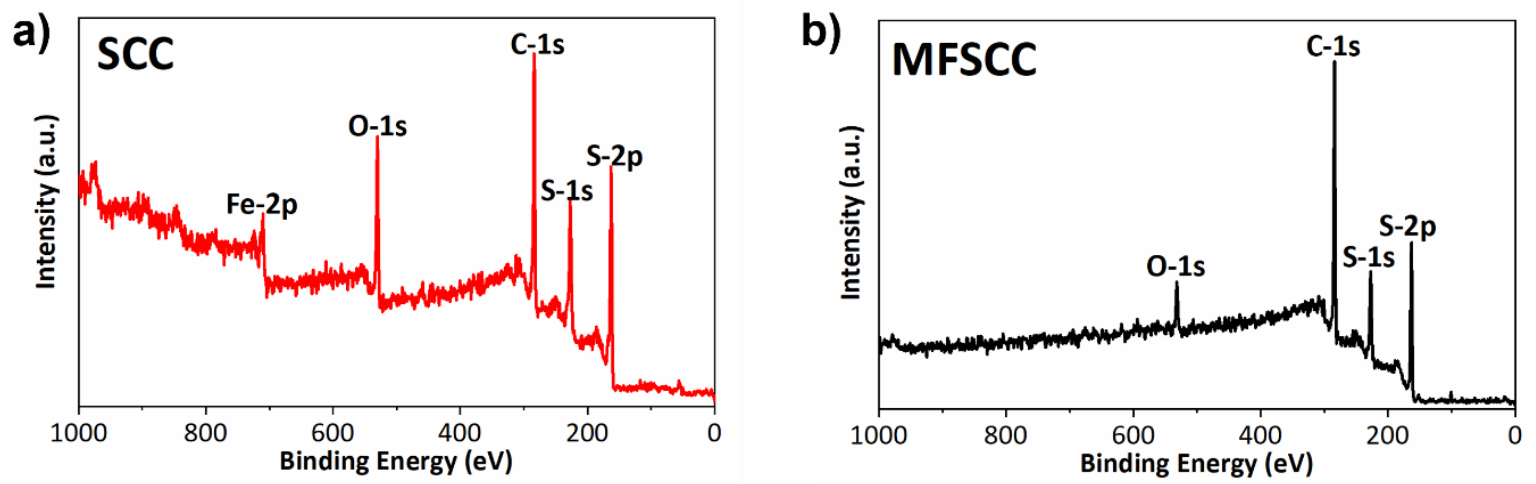

Figure S3. XPS survey spectra of a) SCC and b) MFSCC particulates.
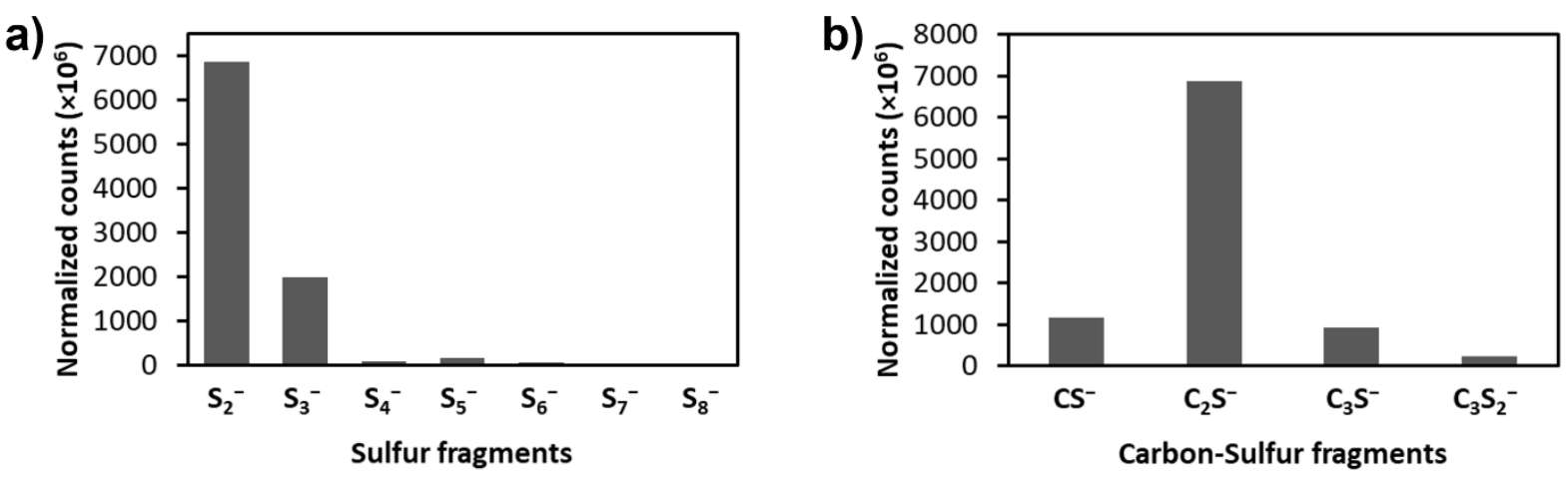

Figure S4. TOF-SIMS distribution of a) sulfur fragment ions and b) carbon/sulfur fragment ions of the SCC composite. 
3. Characterization and electrochemistry of pyrite-type iron disulfide
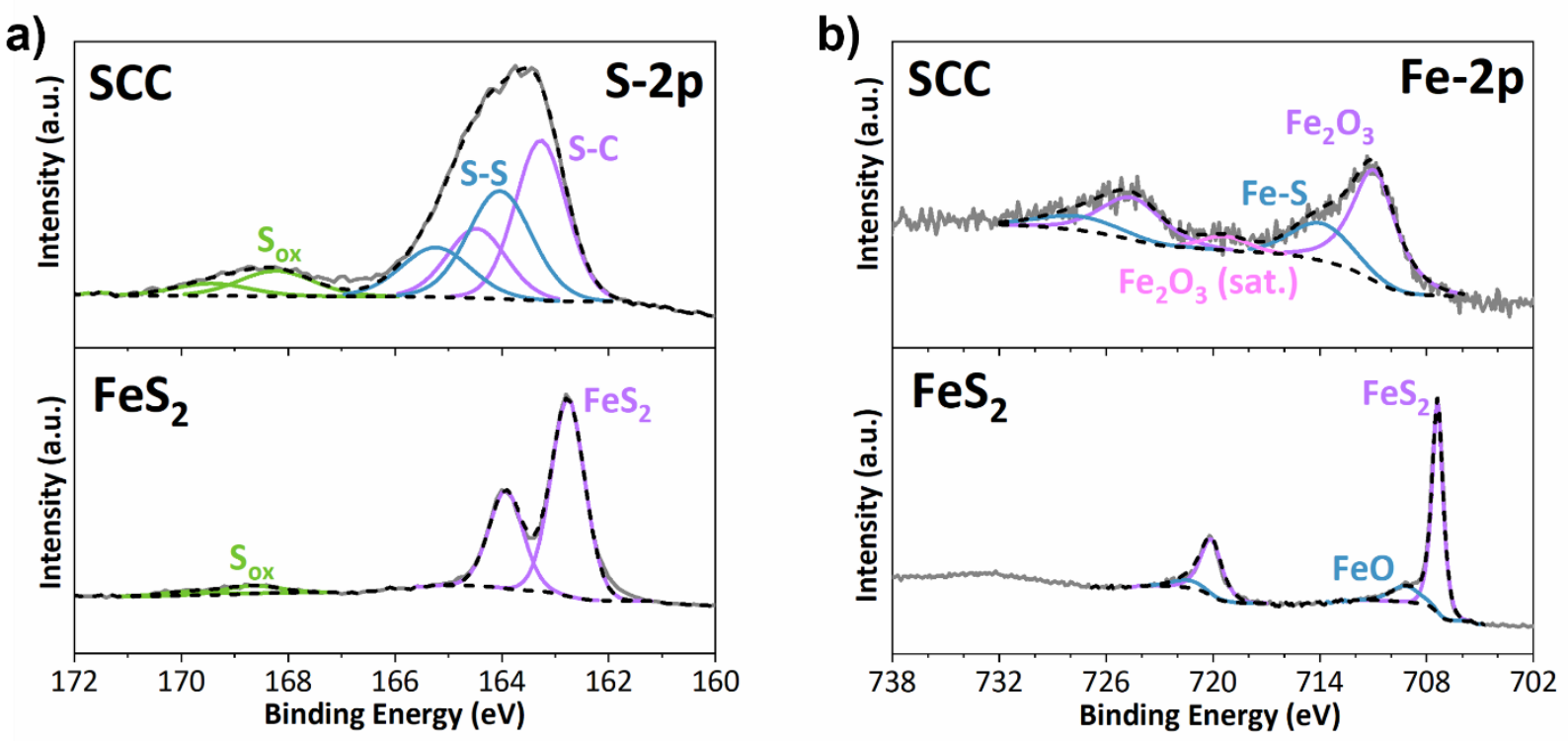

Figure S5. Deconvoluted high resolution a) sulfur-2p (S-2p) and b) iron-2p (Fe-2p) XPS spectra of SCC in comparison to as-purchased pyrite-type iron disulfide $\left(\mathrm{FeS}_{2}\right)$. Commercial pyrite-type $\mathrm{FeS}_{2}$ spectra were in good agreement with previously reported values. ${ }^{3,4}$ 
a)

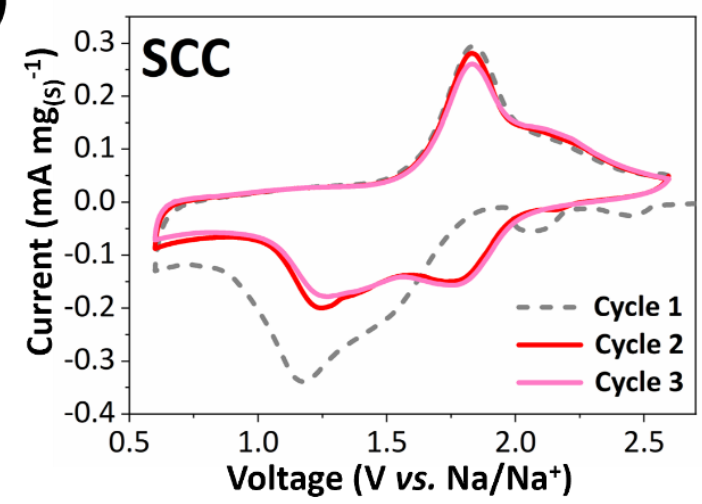

c)

e)

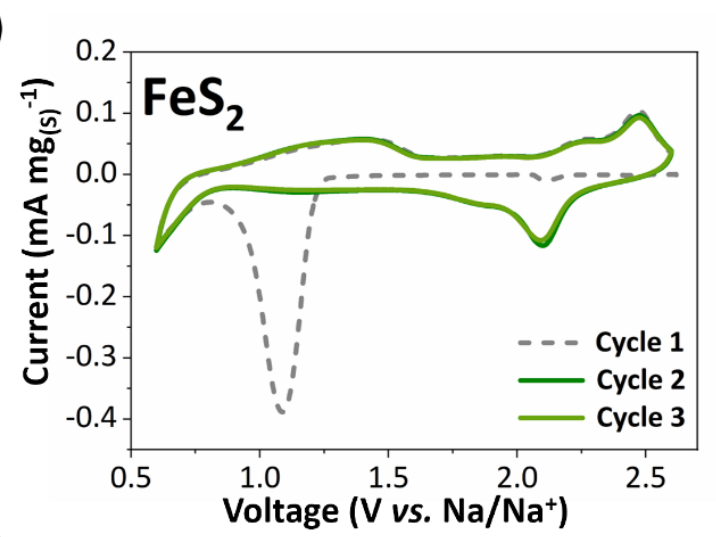

b)

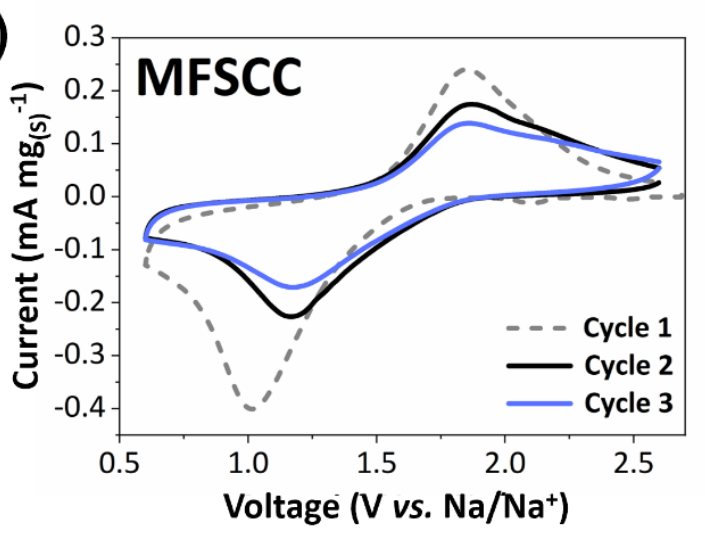

d)

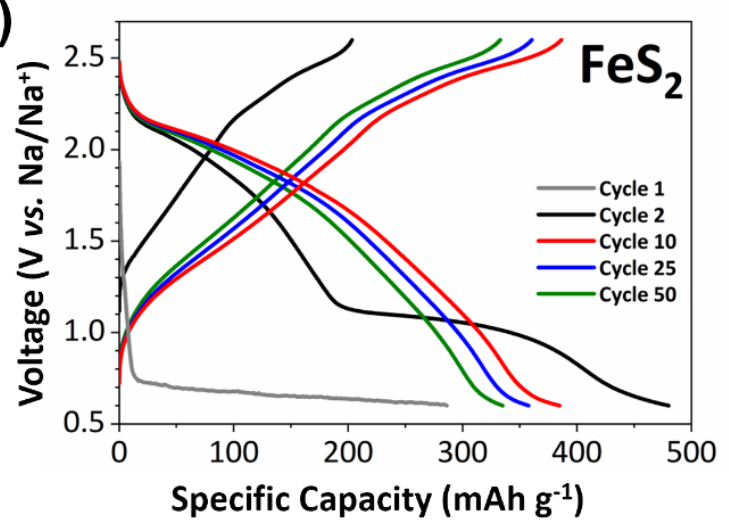

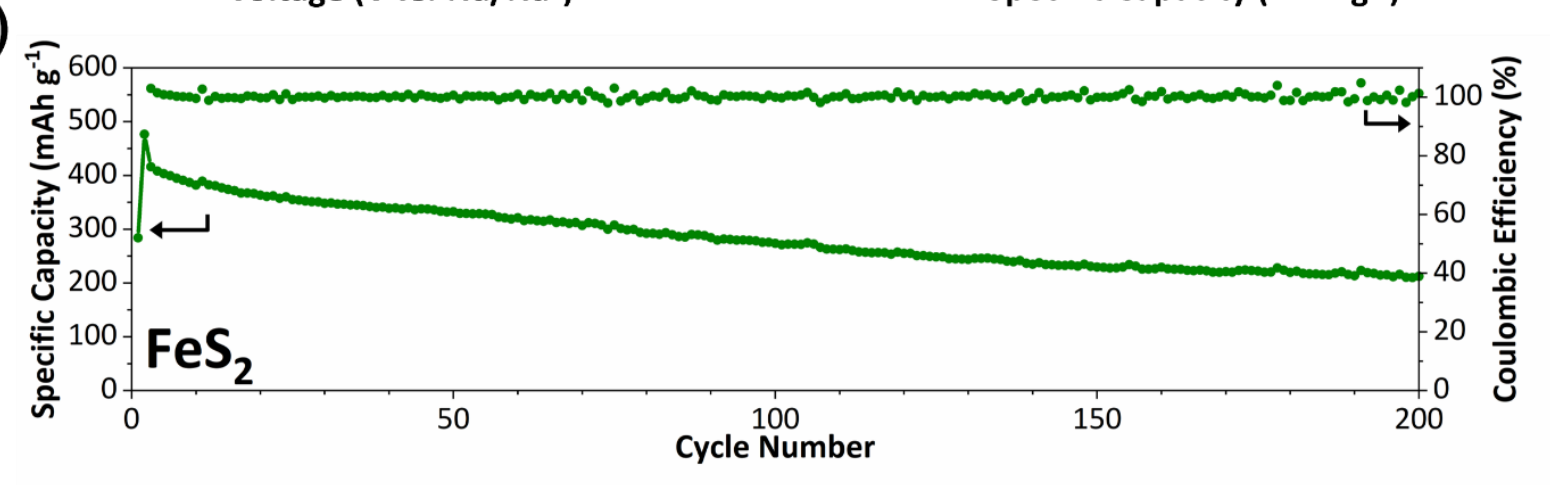

Figure S6. Cyclic voltammograms of the initial three cycles of a) SCC, b) MFSCC and c) commercial pyrite-type $\mathrm{FeS}_{2}$ cathodes obtained with a scan rate of $0.05 \mathrm{mV} \mathrm{s}^{-1}$, starting from open circuit potential to $2.6 \mathrm{~V}$ vs. $\mathrm{Na} / \mathrm{Na}^{+}$. d) Galvanostatic charge/discharge profiles of $\mathrm{FeS} 2$ cathode (mass loading $=0.931 \mathrm{mg} \mathrm{cm}^{-2}$ ) coupled with a sodium metal anode, cycled at $0.2 \mathrm{~A} \mathrm{~g}^{-1}$. Electrolyte: $1 \mathrm{M} \mathrm{NaClO}_{4}$ in $1: 1 v / v$ EC/DMC solvent containing $40 \mathrm{mM} \mathrm{SnCl}_{2}$ and 8 vol.\% $\mathrm{FEC}$ additives. The $\mathrm{FeS}_{2}$ cathodes were prepared with the same procedure as the sulfurized cyclopentadienyl composites. e) Cycling performance and coulombic efficiencies of the $\mathrm{FeS}_{2}$ cell. 


\section{Post-mortem XPS spectra of cycled SCC cathodes}
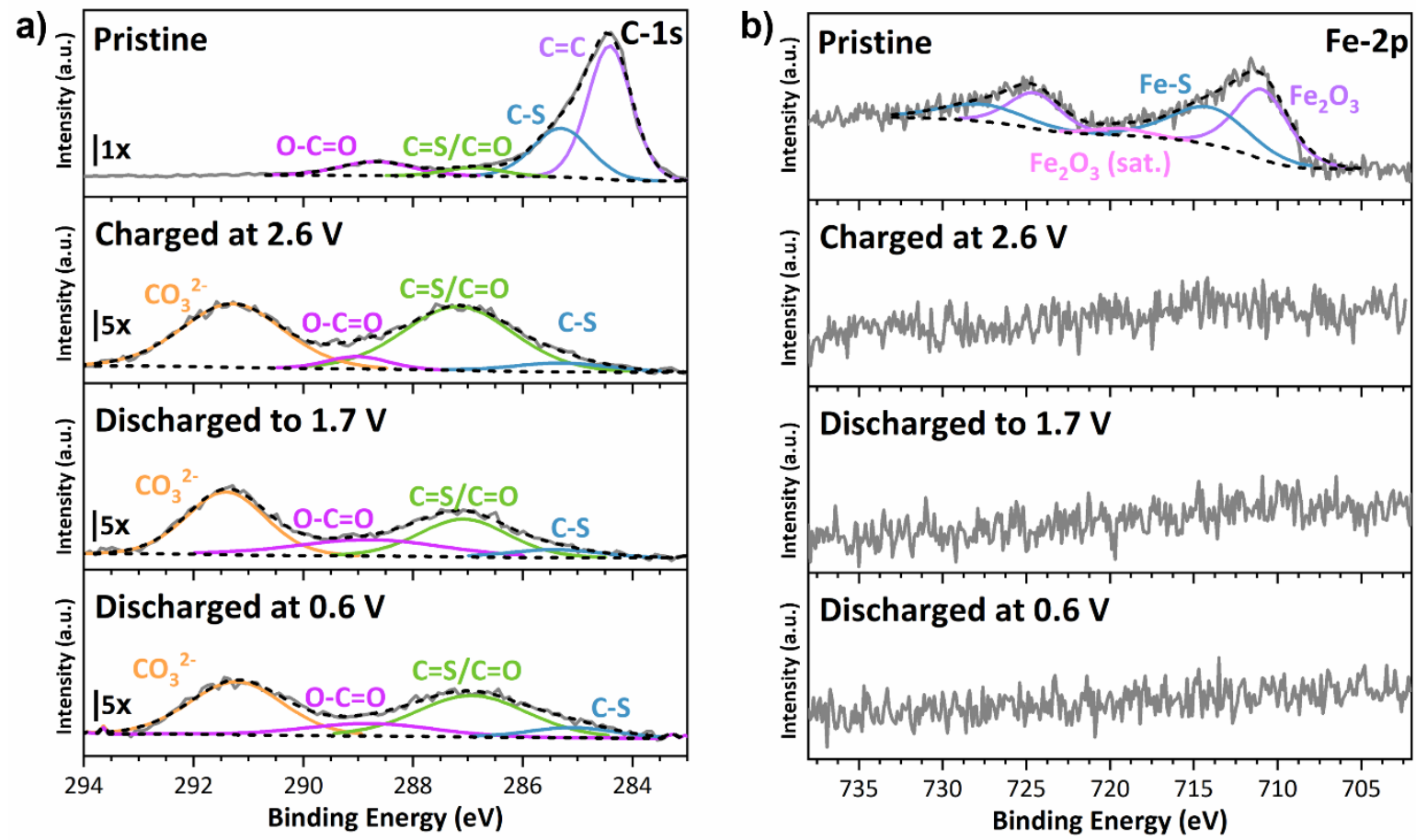

Figure S7. Deconvoluted high resolution a) carbon-1s (C-1s) and b) iron-2p (Fe-2p) ex-situ XPS spectra of SCC cathodes under varying charge/discharge conditions. $\mathrm{CO}_{3}{ }^{2-}$ of the $\mathrm{C}-1 \mathrm{~s}$ spectra arises from solid-electrolyte interphase obtained with the fluoroethylene carbonate additive used. 


\section{Galvanostatic cycling of SCC with lithium metal anode}

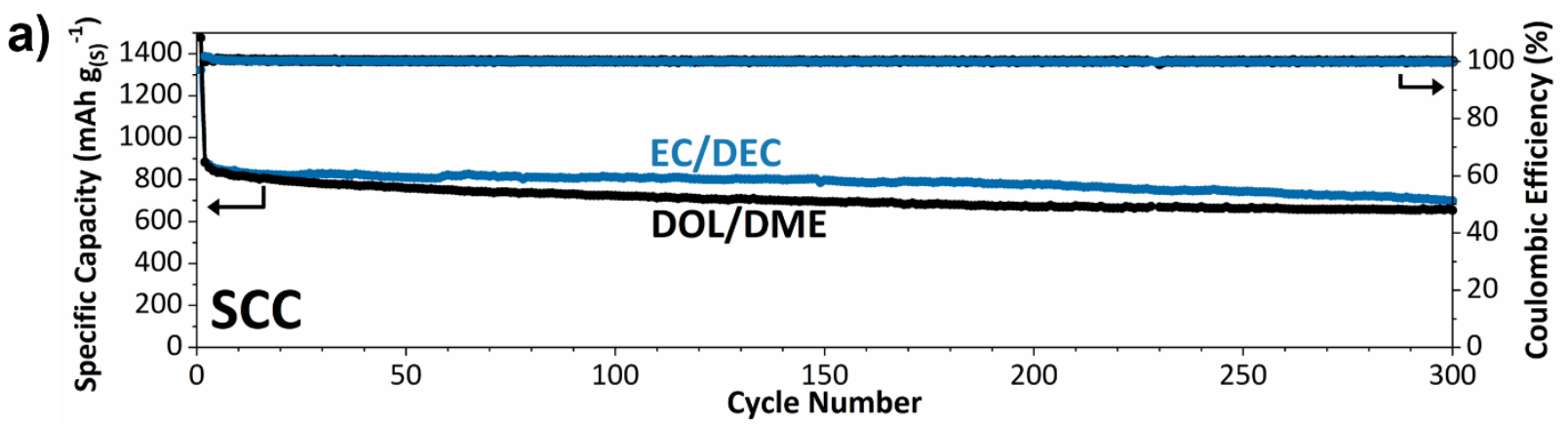

b)

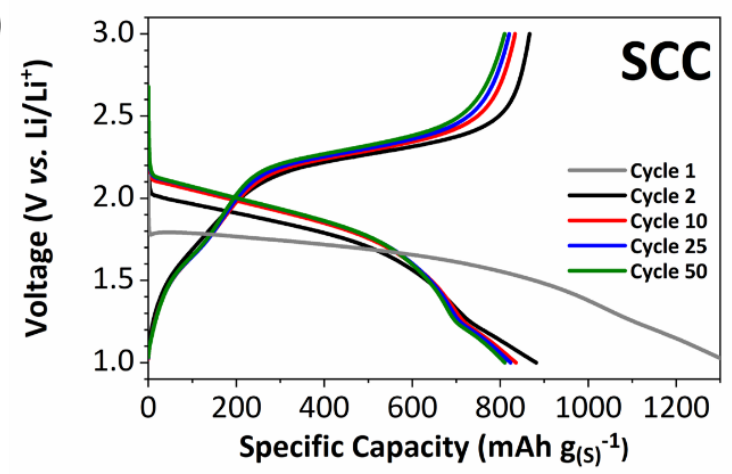

c)

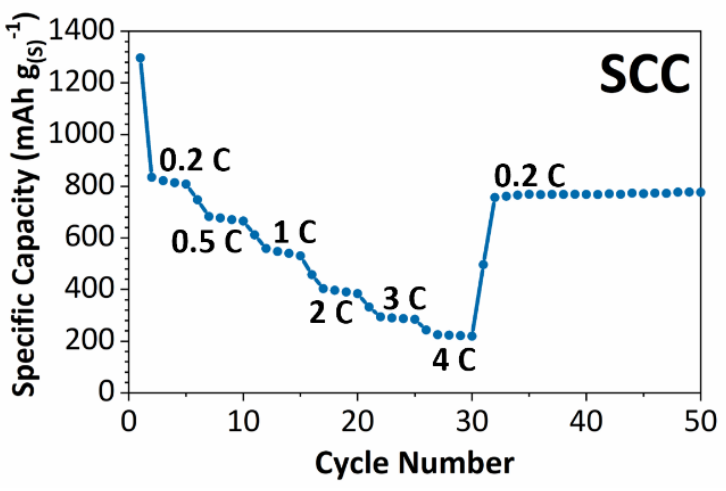

Figure S8. a) Cycling performance of SCC cathodes in a lithium-sulfur cell, with both a carbonatebased electrolyte of $1 \mathrm{M} \mathrm{LiPF}_{6}$ in 1:1 $v / v$ EC/DEC solvent as well as an ether-based, lithium nitratefree electrolyte of $1 \mathrm{M}$ LiTFSI in 1:1 v/v DOL/DME, cycled at $0.2 \mathrm{C}$. b) Galvanostatic charge/discharge profiles at $0.2 \mathrm{C}$ and c) rate performance between $0.2 \mathrm{C}$ and $4 \mathrm{C}$ of SCC for lithium-sulfur cells with carbonate-based electrolyte. 


\section{Comparison of SCC synthesis methods}

As mentioned in Section 1.2 above, SCC was synthesized via two methods, namely a co-melting method and a "mixed-phase" method.
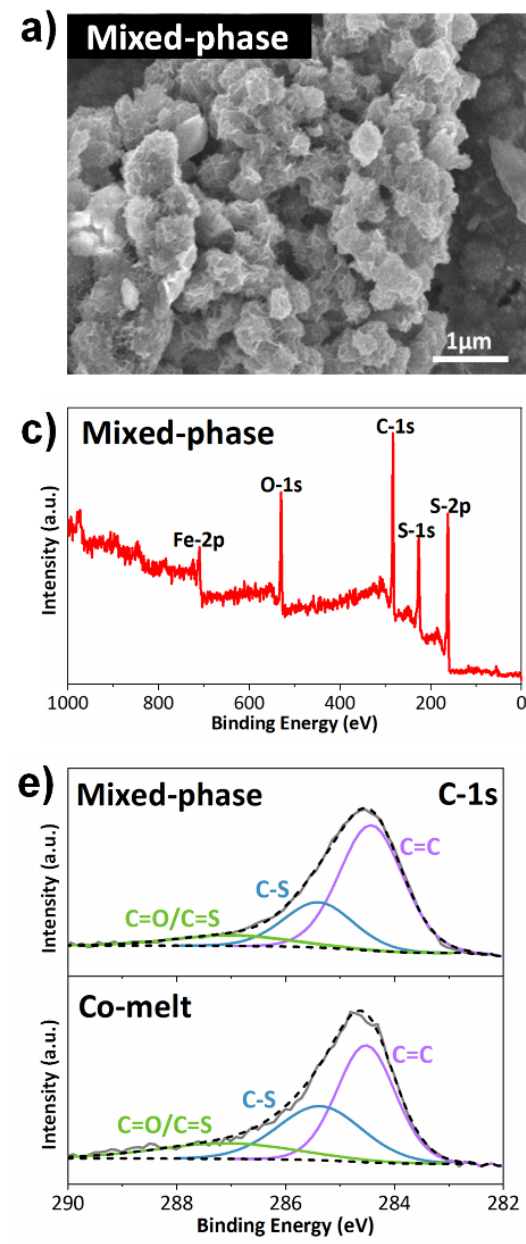
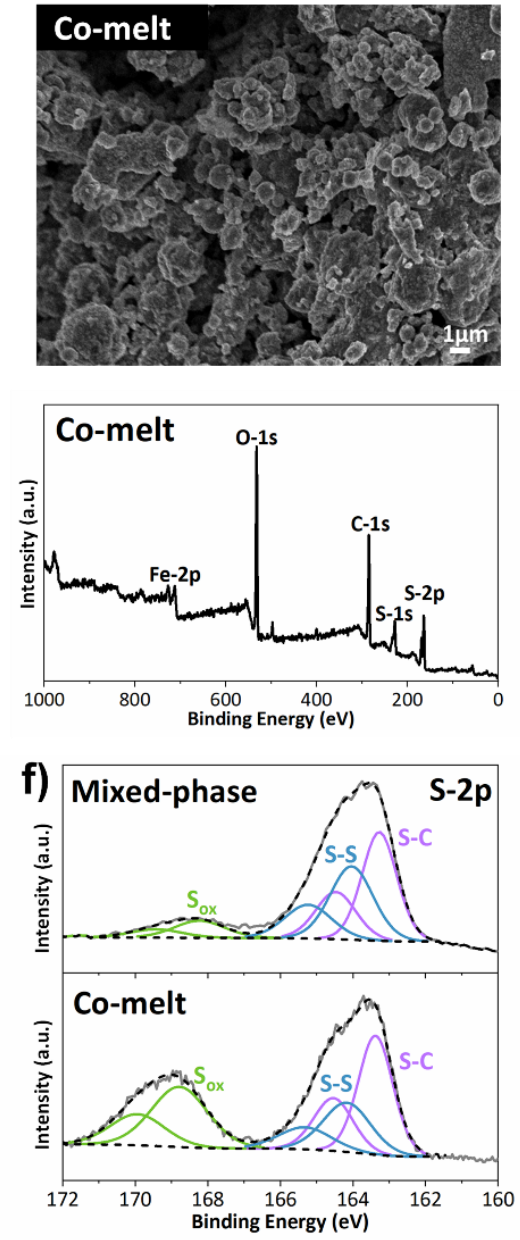

b)

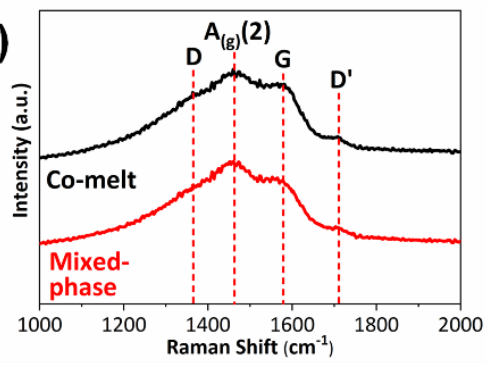

d)
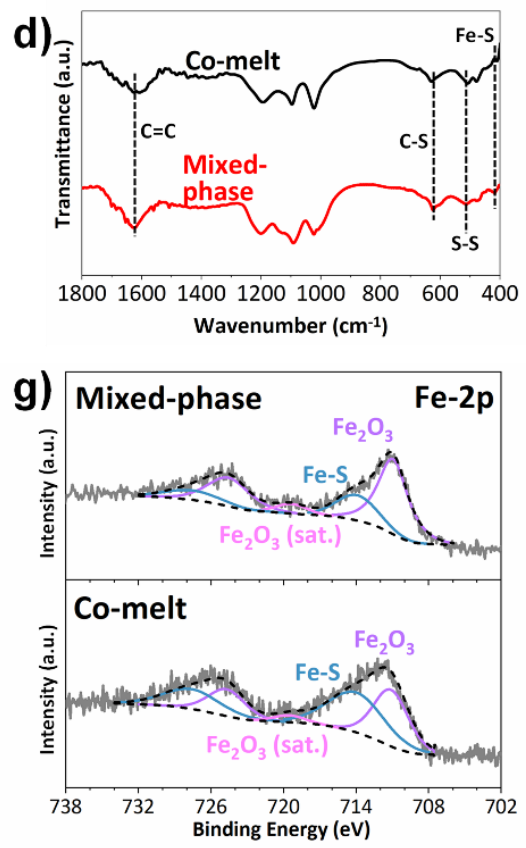

Figure S9. Comparison between a) SEM images, b) Raman spectra and c) XPS survey spectra, d) FTIR spectra and deconvoluted high resolution of e) C-1s; f) S-2p; and g) Fe-2p XPS spectra of SCC synthesised via either co-melting or the mixed phase method. 


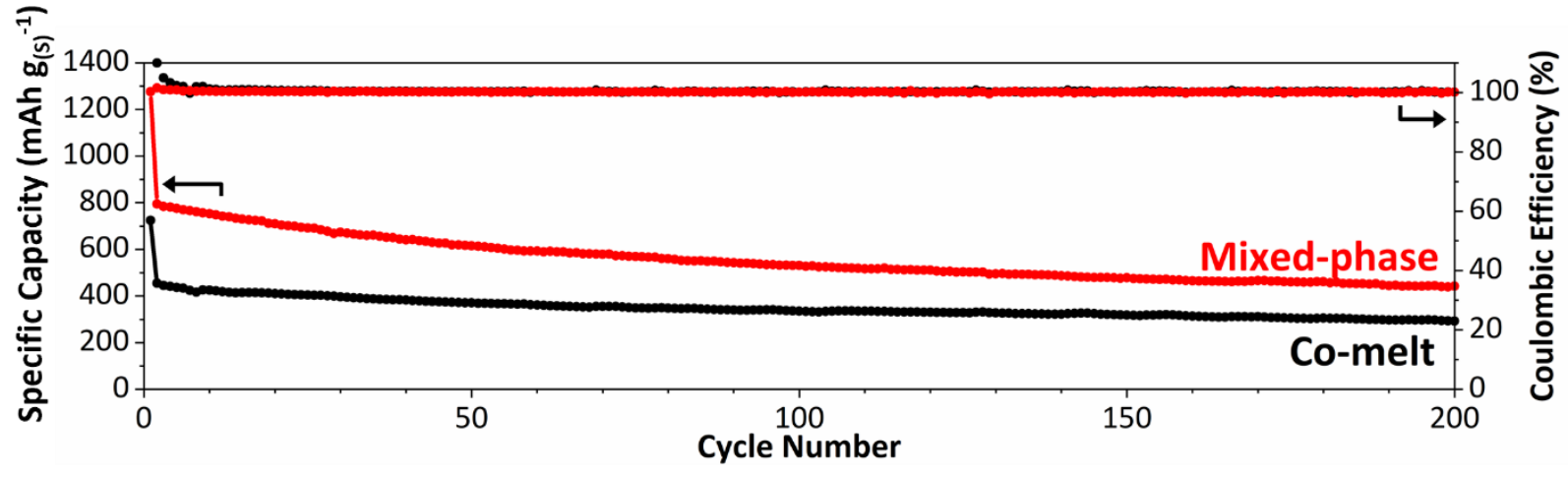

Figure S10. Comparison of cycling performance of SCC cathodes synthesised via the different methods in a sodium-sulfur cell at $0.2 \mathrm{C}$. Electrolyte: $1 \mathrm{M} \mathrm{NaClO}_{4}$ in $1: 1 v / v \mathrm{EC} / \mathrm{DMC}$ solvent containing $40 \mathrm{mM} \mathrm{SnCl}_{2}$ and 8 vol.\% FEC additives. 


\section{Performance Benchmarking for Room-Temperature Sodium-Sulfur Batteries}

Table S3. Comparison of cycling performances and sulfur loadings of recent Na-S battery cathodes based on covalently-bonded sulfur and short-chain sulfur composites.

\begin{tabular}{|c|c|c|c|c|c|}
\hline $\begin{array}{l}\text { Covalently bonded } \\
\text { sulfur and short- } \\
\text { chain sulfur } \\
\text { composite }\end{array}$ & $\begin{array}{l}\text { Initial specific } \\
\text { capacity }(C- \\
\text { rate/ current } \\
\text { density) }\end{array}$ & $\begin{array}{l}\text { Final cycle- } \\
\text { specific } \\
\text { capacity }(C- \\
\text { rate/ current } \\
\text { density) }\end{array}$ & $\begin{array}{l}\text { Max. cycle } \\
\text { life } \\
\text { demonstra } \\
\text { ted }\end{array}$ & $\begin{array}{l}\text { S-content (wt\%) } \\
\text { of composite / } \\
\text { areal loading }\end{array}$ & $\begin{array}{l}\text { Ref. } \\
\text { No. }\end{array}$ \\
\hline $\begin{array}{l}\text { Sulfurized } \\
\text { cyclopentadienyl } \\
\text { composite }\end{array}$ & $\begin{array}{l}795 \mathrm{mAh} \mathrm{g}_{(\mathrm{s})}{ }^{-1} \\
(0.2 \mathrm{C})\end{array}$ & $\begin{array}{l}442 \mathrm{mAh} \mathrm{g}_{(\mathrm{S})}^{-1} \\
(0.2 \mathrm{C})\end{array}$ & 200 & $\begin{array}{l}57.0-57.2 \% / \\
0.5-0.8 \mathrm{mg}_{(\mathrm{s})} \mathrm{cm}^{-2}\end{array}$ & $\begin{array}{l}\text { This } \\
\text { work }\end{array}$ \\
\hline \multicolumn{6}{|c|}{ - Sulfur co-polymers } \\
\hline $\begin{array}{l}\text { Thioether- } \\
\text { functionalized } \\
\text { carbon }\end{array}$ & $\begin{array}{l}\sim 600 \mathrm{mAh} \mathrm{g}_{(\mathrm{s})}{ }^{-1} \\
\left(0.1 \mathrm{~A} \mathrm{~g}^{-1}\right)\end{array}$ & $\begin{array}{l}330 \mathrm{mAh} \mathrm{g}_{(\mathrm{s})}^{-1} \\
\left(1 \mathrm{~A} \mathrm{~g}^{-1}\right)\end{array}$ & 800 & $\begin{array}{l}21.5 \% / \\
0.9-1.1 \mathrm{mg} \mathrm{cm}^{-2} \\
\left(\sim 0.19-0.24 \mathrm{mg}_{(\mathrm{S})}\right. \\
\left.\mathrm{cm}^{-2}\right)\end{array}$ & 5 \\
\hline $\begin{array}{l}\text { Benzenedisulfonic } \\
\text { acid/sulfate-based } \\
\text { sulfurized carbon }\end{array}$ & $\begin{array}{l}696 \mathrm{mAh} \mathrm{g}_{(\mathrm{s})}{ }^{-1} \\
\left(2.5 \mathrm{~A} \mathrm{~g}^{-1}\right)\end{array}$ & $\begin{array}{l}452 \mathrm{mAh} \mathrm{g}_{(\mathrm{s})}^{-1} \\
\left(2.5 \mathrm{~A} \mathrm{~g}^{-1}\right)\end{array}$ & 1000 & $\begin{array}{l}40.1 \% / \\
\sim 3 \mathrm{mg} \mathrm{cm}^{-2}(\sim 1.20 \\
\left.\mathrm{mg}_{(\mathrm{S})} \mathrm{cm}^{-2}\right)\end{array}$ & 6 \\
\hline $\begin{array}{l}\text { Benzoxazine- } \\
\text { derived sulfur- } \\
\text { copolymer on } \\
\text { reduced graphene } \\
\text { oxide }\end{array}$ & $\begin{array}{l}\sim 500 \mathrm{mAh} \mathrm{g} \mathrm{g}^{-1} \\
\left(1 \mathrm{~A} \mathrm{~g}^{-1}\right)\end{array}$ & $\begin{array}{l}285 \mathrm{mAh}^{-1} \\
\left(1 \mathrm{~A} \mathrm{~g}^{-1}\right)\end{array}$ & 100 & $\begin{array}{l}\sim 89 \% / \\
\sim 2.14 \mathrm{mg} \mathrm{cm}^{-2}\end{array}$ & 7 \\
\hline \multicolumn{6}{|c|}{ - Other covalently bonded sulfur and short-chain sulfur cathodes } \\
\hline $\begin{array}{l}\text { Polymer of intrinsic } \\
\text { microporosity, } \\
\text { PIM-1-based } \\
\text { carbon-sulfur } \\
\text { composite }\end{array}$ & $\begin{array}{l}\sim 500 \mathrm{mAh} \mathrm{g}^{-1} \\
(0.1 \mathrm{C})\end{array}$ & $\begin{array}{l}\sim 550 \mathrm{mAh} \mathrm{g}^{-1} \\
(0.1 \mathrm{C})\end{array}$ & 250 & $\begin{array}{l}\sim 30 \% / \\
\sim 0.41 \mathrm{mg}_{(\mathrm{s})} \mathrm{cm}^{-2}\end{array}$ & 8 \\
\hline $\begin{array}{l}\text { Sulfurized } \\
\text { polyacrylonitrile } \\
\text { particles }\end{array}$ & $\begin{array}{l}1195 \mathrm{mAh} \mathrm{g}_{(\mathrm{s})}{ }^{-1} \\
(0.5 \mathrm{C})\end{array}$ & $\begin{array}{l}1000 \mathrm{mAh} \mathrm{g}_{(\mathrm{s})}{ }^{-1} \\
(0.5 \mathrm{C})\end{array}$ & 1000 & $\begin{array}{l}41 \% / \sim 0.5-0.7 \\
\mathrm{mg}_{(\mathrm{s})} \mathrm{cm}^{-2}\end{array}$ & 9 \\
\hline $\begin{array}{l}\text { Sulfurized } \\
\text { polyacrylonitrile } \\
\text { fibres }\end{array}$ & $\begin{array}{l}219 \mathrm{mAh} \mathrm{g}^{-1} \\
(0.1 \mathrm{C})\end{array}$ & $\begin{array}{l}153 \mathrm{mAh} \mathrm{g}^{-1} \\
(1 \mathrm{C})\end{array}$ & 500 & $\begin{array}{l}31.4 \% / \sim 1.0-1.2 \\
\mathrm{mg}(\sim 0.31-0.38 \\
\mathrm{mg}_{(\mathrm{s})} \mathrm{cm}^{-2}\end{array}$ & 10 \\
\hline $\begin{array}{l}\text { Carbonized ZIF-8 } \\
\text { microporous } \\
\text { carbon-sulfur } \\
\text { composite }\end{array}$ & $\begin{array}{l}1137 \mathrm{mAh} \mathrm{g}_{(\mathrm{s})}{ }^{-1} \\
(0.5 \mathrm{C})\end{array}$ & $\begin{array}{l}980 \mathrm{mAh} \mathrm{g}_{(\mathrm{s})}{ }^{-1} \\
(0.5 \mathrm{C})\end{array}$ & 800 & $\begin{array}{l}29.4-34.0 \% / 0.5- \\
0.7 \mathrm{mg}_{(\mathrm{s})} \mathrm{cm}^{-2}\end{array}$ & 11 \\
\hline
\end{tabular}




\section{References}

1. Li, X.; Liang, J.; Li, W.; Luo, J.; Li, X.; Yang, X.; Hu, Y.; Xiao, Q.; Zhang, W.; Li, R.; Sham, T.-K.; Sun, X., Stabilizing Sulfur Cathode in Carbonate and Ether Electrolytes: Excluding Long-Chain Lithium Polysulfide Formation and Switching Lithiation/Delithiation Route. Chem. Mater. 2019, 31 (6), 2002-2009.

2. Velasco, S.; Román, F. L.; White, J. A., On the Clausius-Clapeyron Vapor Pressure Equation. J. Chem. Educ. 2009, 86 (1), 106.

3. Karthe, S.; Szargan, R.; Suoninen, E., Oxidation of pyrite surfaces: a photoelectron spectroscopic study. Appl. Surf. Sci. 1993, 72 (2), 157-170.

4. Nesbitt, H. W.; Bancroft, G. M.; Pratt, A. R.; Scaini, M. J., Sulfur and iron surface states on fractured pyrite surfaces. Am. Mineral. 1998, 83 (9-10), 1067-1076.

5. Chen, K.; Li, H.; Xu, Y.; Liu, K.; Li, H.; Xu, X.; Qiu, X.; Liu, M., Untying thioether bond structures enabled by "voltage-scissors" for stable room temperature sodium-sulfur batteries. Nanoscale 2019, 11 (13), 5967-5973.

6. Wu, T.; Jing, M.; Yang, L.; Zou, G.; Hou, H.; Zhang, Y.; Zhang, Y.; Cao, X.; Ji, X., Controllable Chain-Length for Covalent Sulfur-Carbon Materials Enabling Stable and HighCapacity Sodium Storage. Adv. Energy Mater. 2019, 9 (9), 1803478.

7. Ghosh, A.; Shukla, S.; Monisha, M.; Kumar, A.; Lochab, B.; Mitra, S., Sulfur Copolymer: A New Cathode Structure for Room-Temperature Sodium-Sulfur Batteries. ACS Energy Lett. 2017, 2 (10), 2478-2485.

8. Jeon, J. W.; Kim, D.-M.; Lee, J.; Lee, J.-C.; Kim, Y. S.; Lee, K. T.; Kim, B. G., PIM1-based carbon-sulfur composites for sodium-sulfur batteries that operate without the shuttle effect. J. Mater. Chem. A 2020, 8 (7), 3580-3585.

9. $\quad$ Eng, A. Y. S.; Nguyen, D.-T.; Kumar, V.; Subramanian, G. S.; Ng, M.-F.; Seh, Z. W., Tailoring binder-cathode interactions for long-life room-temperature sodium-sulfur batteries. $J$. Mater. Chem. A 2020, 8 (43), 22983-22997.

10. Hwang, T. H.; Jung, D. S.; Kim, J.-S.; Kim, B. G.; Choi, J. W., One-Dimensional Carbon-Sulfur Composite Fibers for Na-S Rechargeable Batteries Operating at Room Temperature. Nano Lett. 2013, 13 (9), 4532-4538.

11. Eng, A. Y. S.; Wang, Y.; Nguyen, D.-T.; Tee, S. Y.; Lim, C. Y. J.; Tan, X. Y.; Ng, M.-F.; Xu, J.; Seh, Z. W., Tunable Nitrogen-Doping of Sulfur Host Nanostructures for Stable and Shuttle-Free Room-Temperature Sodium-Sulfur Batteries. Nano Lett. 2021, 21 (12), 54015408. 